\title{
Does texting while walking really affect gait in young adults?
}

Valentina Agostini ${ }^{1 *}$, Francesco Lo Fermo ${ }^{2}$, Giuseppe Massazza ${ }^{3}$ and Marco Knaflitz ${ }^{1}$

\begin{abstract}
Background: Texting on a smartphone while walking has become a customary task among young adults. In recent literature many safety concerns on distracted walking have been raised. It is often hypothesized that the allocation of attentional resources toward a secondary task can influence dynamic stability. In the double task of walking and texting it was found that gait speed is reduced, but there is scarce evidence of a modified motor control strategy compromising stability. The aim of this study is twofold: 1) to comprehensively examine the gait modifications occurring when texting while walking, including the study of the lower limb muscle activation patterns, 2) to specifically assess the co-contraction of ankle antagonist muscles. We hypothesized that texting while walking increases co-contractions of ankle antagonist muscles when the body weight is transferred from one lower limb to the other, to improve the distal motor control and joint stabilization.
\end{abstract}

Methods: From the gait data collected during an instrumented walk lasting $3 \mathrm{~min}$, we calculated the spatio-temporal parameters, the ankle and knee kinematics, the muscle activation patterns of tibialis anterior, gastrocnemius lateralis, peroneus longus, rectus femoris, and lateral hamstrings, and the co-contraction (occurrence and duration) of the ankle antagonist muscles (tibialis anterior and gastrocnemius lateralis), bilaterally.

Results: Young adults showed, overall, small gait modifications that could be mainly ascribable to gait speed reduction and a modified body posture due to phone handling. We found no significant alterations of ankle and knee kinematics and a slightly delayed activation onset of the left gastrocnemius lateralis. However, we found an increased co-contraction of tibialis anterior and gastrocnemius lateralis, especially during mid-stance. Conversely, we found a reduced co-contraction during terminal stance.

Conclusions: Our results suggest that, in young adults, there is an adjustment of the motor control strategy aimed at increasing ankle joint stability in a specific and "critical" phase of the gait cycle, when the body weight is transferred from one leg to the other.

Keywords: Gait, Gait analysis, Texting and walking, Muscle co-contraction, EMG, Smartphone use, Dual task

\section{Background}

Young individuals rarely just walk. They are frequently engaged in additional tasks, such as talking on a mobile phone, listening to music or texting messages. Emerging research evidenced the dangers of distracted walking and reduced situation awareness in pedestrians using smartphones [1]. In particular, it was reported that texting on a smartphone creates a significantly greater interference effect on walking than talking or reading $[2,3]$. As a matter of fact, the activity of texting while walking

\footnotetext{
* Correspondence: valentina.agostini@polito.it

'Dipartimento di Elettronica e Telecomunicazioni, Politecnico di Torino, Corso Duca degli Abruzzi 24, 10129 Torino, Italy

Full list of author information is available at the end of the article
}

is a more complex task, since it usually integrates visualmotor coordination, bimanual movements for tapping with thumbs of both hands, and cognitive attention to the message content. A recent study showed that, for what concerns their frontal plane margin of stability, experienced texters are more affected by the physical than by the cognitive demand of texting [4]. Subjects may try to control foot placement and joint kinematics during cell phone use or another cognitive task with a visual component, to ensure sufficient dynamic margins of stability [5].

Existing research provided insight into spatio-temporal parameter modifications of texting while walking and, 
usually, a reduced gait speed was reported $[2,3,6,7]$. Furthermore, stride-to-stride variability was found to be increased in several dual task experiments involving cognitive-demanding tasks [8-10]. However, writing on a smartphone while walking involves both cognitive and physical resources, the integration of gross and fine motor functions, near and far vision. Hence, stride-to-stride variability might be even further increased.

Previous research provided evidence that individuals, while texting, have altered head and trunk kinematics [3], since their head is almost inevitably inclined forwards to read the display. However, little is known on how the Central Nervous System (CNS) adapts to control lower limbs and increase stability, and to what extent, and how, young adults modify their motor scheme during the dual task of texting and walking. More specifically, none of the existing studies reported gait adaptations in terms of ankle and knee joint kinematics, lower limb muscle activation patterns, and co-contraction of ankle antagonist muscles.

Recent literature on the detection of muscle activation timing from the surface electromyographic (EMG) signal highlighted the importance of using innovative methods, known under the name of "statistical gait analysis", to properly handle the large intra- and inter-subject variability of human gait [11-15]. These methods may constitute a valuable analysis tool when small changes in the muscle activation patterns are expected [13, 15], as it may happen in dual-task protocols evaluating the walking function with and without some additional task. However, to the best of our knowledge, they have never been applied within this context.

Muscle co-contraction is the simultaneous activation of agonist and antagonist muscles crossing a joint [16] and its function is to increase joint stiffness. A recent study on young adults showed that tibialis anterior (TA) and gastrocnemius lateralis (GL) act as pure agonist/ antagonists for ankle plantar/dorsiflexion (no cocontraction) in only $21 \%$ of strides [17]. In the remaining strides, co-contractions appeared, both in stance and/or swing, with the probable function of improving balance and control ankle stability. It is known that attentional resources toward a secondary cognitive task can lead to a diminished ankle proprioceptive performance [18]. Hence, we hypothesized that texting while walking increases co-contractions of ankle antagonist muscles when the body weight is transferred from one lower limb to the other, to improve the distal motor control and joint stabilization.

The purpose of this study was to comprehensively examine, in a population of young adults, the gait modifications due to texting on a smartphone while walking, with a focus on distal motor control. Along with spatiotemporal parameters and stride-to-stride variability, we analyzed, bilaterally: 1) ankle and knee kinematics, 2) the muscle activation patterns of five lower limb muscles, 3 ) the co-contraction of TA/GL muscles.

\section{Methods}

\section{Participants}

Eighteen healthy young adults, aged from 20 to 30 years, with normal or corrected-to-normal vision, were recruited from the university community ( 8 males/ 10 females, height: $1.69 \pm 0.08 \mathrm{~m}$; weight: $63.3 \pm 10 \mathrm{~kg}$ ). Participants were eligible if they used, on a daily basis, a smartphone with a display between 3.5 and 5 inches, with a touch screen and virtual QWERTY keyboard, and had more than 2 months experience with their current phone. Individuals reporting neurological, musculoskeletal disorders or other conditions that could affect their gait or capacity of typing were excluded from the study.

This study was approved by the local Institutional Review Board and all procedures conformed to the Helsinki declaration. Written informed consent was obtained by all participants.

\section{Procedures}

Participants were assessed in a well-lit room, over a straight path of $15 \mathrm{~m}$. Subjects were asked to walk back and forth along the path, at their natural pace, for $3 \mathrm{~min}$ (Fig. 1). We examined 2 different conditions: a) walking, b) walking and texting. The two conditions were administered randomly. In condition b) no instruction was given on task prioritization to better reproduce a realworld situation. Participants used their own smartphone and their usual typing method (one or two hands). They were asked to type a message describing their own activities on the day before the test. After the test completion, they were asked to send the message to the experimenter, so that he could count the total number of characters written during the $3 \mathrm{~min}$, in order to

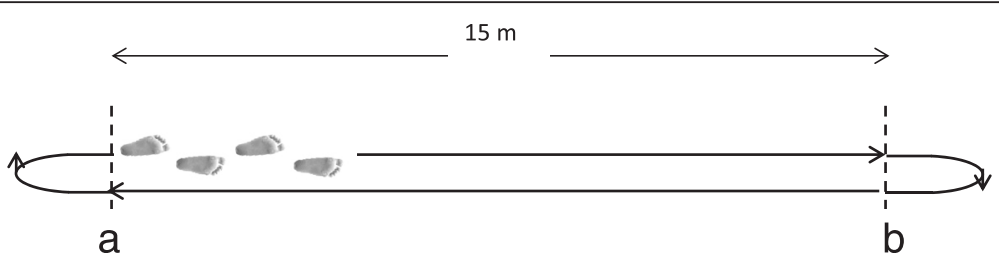

Fig. 1 Walking path. Subjects are instructed to pass the marks $(\mathbf{a}, \mathbf{b})$ before decelerating and turning back 
estimate the average typing speed, calculated as the number of characters per minute.

The experimenter timed each subject's passage through the $15-\mathrm{m}$ walkway (see Fig. 1), with the exclusion of direction changes. More specifically, he measured the time that the subject walked from $A$ to $B$, then from B to A, then from A to B again, etc..... Gait speed stability among the different A-B passages was checked and the average gait speed was defined as the total distance walked in a straight line divided by the total time required to go through it.

Subjects walked barefooted, with thin foot-switches placed under the foot-soles (size: $10 \mathrm{~mm} \times 10 \mathrm{~mm} \times$ $0.5 \mathrm{~mm}$; activation force: $3 \mathrm{~N}$ ), beneath the first and fifth metatarsal heads, and beneath the back portion of the heel. Sagittal plane electro-goniometers were placed at ankle and knee joints (accuracy: $0.5 \mathrm{deg}$ ). Surface EMG probes were placed over tibialis anterior (TA), gastrocnemius lateralis (GL), peroneus longus (PL), rectus femoris (RF), and lateral hamstring (LH), bilaterally. EMG probes were active and utilized Agdisks (diameter: 4-mm, inter-electrode distance: $12 \mathrm{~mm}$ ). The signal amplifier had a gain of 1000 and a 3-dB bandwidth from $10 \mathrm{~Hz}$ to $400 \mathrm{~Hz}$. The sampling frequency was $2 \mathrm{kHz}$ and the signals were converted by a 12-bit analog to digital converter. Signals detected by sensors on the subject and a synchronized digital video were recorded by the system STEP32, Medical Technology - DemItalia (Italy).
Since in correspondence of the turns participants had to decelerate, change directions, and reinitiate a forward directed trajectory that involved an acceleration phase, the strides corresponding to direction changes were automatically removed by the system software.

\section{Data analysis}

In each test condition, for each patient, an average of $157 \pm 11$ gait cycles were analyzed. For each lower limb, time events were identified using a 4-level footswitch signal, coded as follows: 1) heel footswitch closed, 2) heel- and (at least one) forefoot-switch also closed, 3) at least one forefoot switch closed, 4) no footswitches closed [19]. The following gait phases were determined: heel contact $(\mathrm{H})$, flat-foot contact $(\mathrm{F})$, push-off/heel-off (P) and swing (Fig. 2). We calculated the duration of the sub-phases of stance H, F, P expressed as percentage of the gait cycle (\% GC).

The stride-to-stride variability was assessed by the coefficient of variation $(\mathrm{CV})$ of the stride time, defined as follows:

$$
\text { CV of stride time }(\%)=\frac{\text { standard deviation }(\text { stride time })}{\text { mean }(\text { stride time })} \cdot 100
$$

Dual task effect (DTE) on gait parameters was calculated as the relative change in performance in the dualtask condition compared to single-task performance:

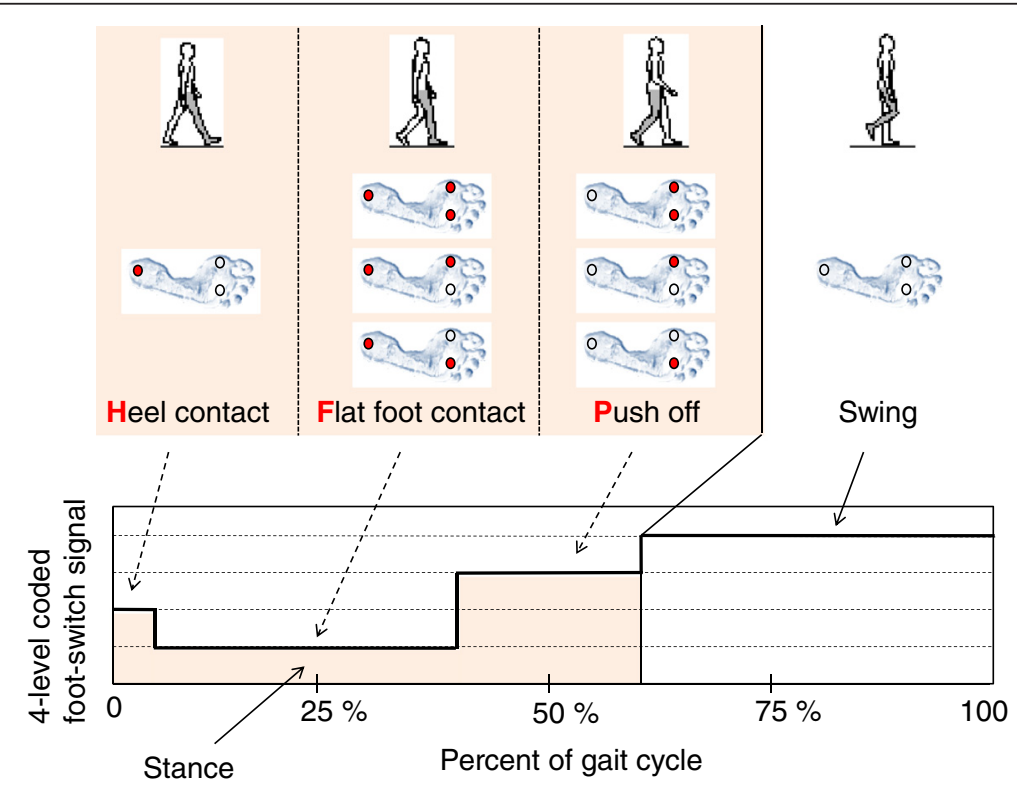

Fig. 2 Gait phases. Foot-switch signal coding (right foot). A red circle under the foot sole indicates a closed foot-switch. The signal has 4 quantization levels: 1) only the heel foot-switch is closed (Heel contact), 2) the heel foot-switch is closed, and at least one of the foot-switches under the forefoot is also closed (Flat foot contact), 3) the heel foot-switch is open, and at least one of the foot-switches under the forefoot is closed (Push off), 4) all the foot-switches are open (Swing) 


$$
D T E=\frac{\mid \text { single task-dual task } \mid}{\text { single task }} \cdot 100
$$

EMG signals were high-pass filtered (cut-off frequency of $20 \mathrm{~Hz}$ ) and then processed by a double-threshold statistical detector [20], embedded in the Step32 system, that provided the onset and offset time instants of muscle activity in a completely user-independent way. This detector was applied to the raw EMG signal and, hence, it did not require any envelope detection (Fig. 3). The detection technique consisted of selecting a first threshold $\zeta$ and observing $m$ successive samples: if at least $r_{0}$ out of successive $m$ samples were above the first threshold $\zeta$, the presence of the signal was acknowledged. In this approach, the second threshold was represented by $r_{0}$. Thus, the behavior of the double-threshold detector was determined by three parameters: the first threshold $\zeta$, the second threshold $\mathrm{r}_{0}$, and the length of the observation window $m$. Their values were selected to jointly minimize the value of false-alarm probability and maximize probability of detection for each specific signal-to-noise ratio. The setting of the first threshold, $\zeta$, was based on the assessment of the background noise level, as a necessary input parameter. Furthermore, the double-threshold detector required to estimate the signal-to-noise ratio in order to fine tune the second threshold, $r_{0}$. The values of the background noise level and the signal-to-noise ratio, necessary to run the double-threshold algorithm, were estimated for each signal by Step32 system, using the statistical approach described in [21]. The length duration of the observation window, $m$, was set equal to $30 \mathrm{~ms}$, that was considered a suitable value for the study of muscle activation in gait analysis [20].

The co-contraction of ankle joint muscles was assessed calculating: 1) the percentage of cycles showing a simultaneous activation of TA and GL, within a specific gait phase (H, F, P and swing), 2) the average co-contraction duration in these cycles (TA/GL simultaneous activation expressed as \% GC).

The EMG activation patterns of TA, GL, PL, RF, and LH, bilaterally, were obtained in the two testing conditions of a) walking and b) walking and texting. In previous studies we found that human locomotion is not characterized by a single "preferred" pattern of muscle activation, but rather by up to 4-5 distinct EMG patterns, each distinguished by a different number of activation intervals occurring within a gait cycle [12, 13]. As an example, in Fig. 3, three different activation patterns of GL were displayed, observed in three different strides extracted from the same walk, showing 1, 2 and 3 activations, respectively. With this example, we wanted to clarify that EMG variability must be properly handled, and that it might be incorrect to apply ensemble averages over EMG patterns showing a different number of activation intervals. Hence, the muscle activation timing was averaged across the various strides of a subject's gait, bundling together only EMG patterns sharing the same number of activation intervals within the gait cycle. EMG patterns sharing the same number of activation intervals were named "activation modalities" [12]. To evaluate the

(a) One activation

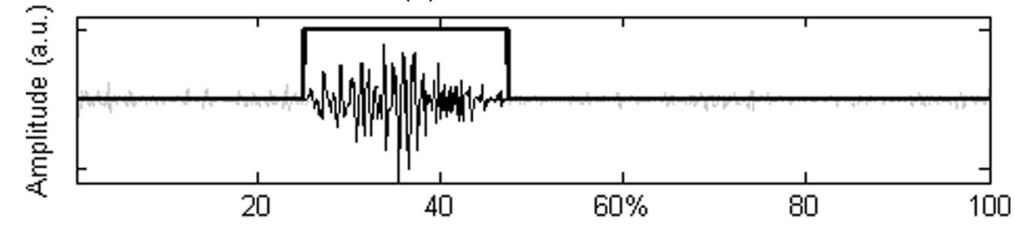

(b) Two activations

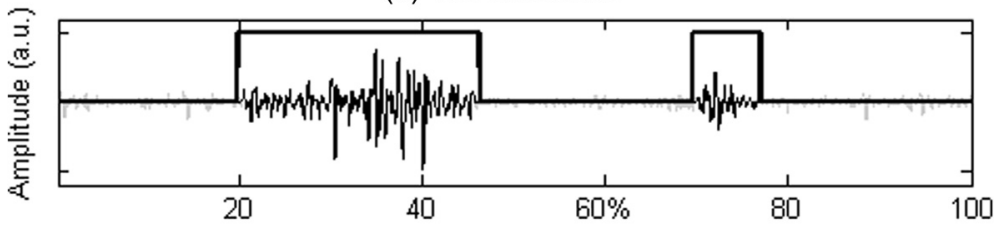

(c) Three activations

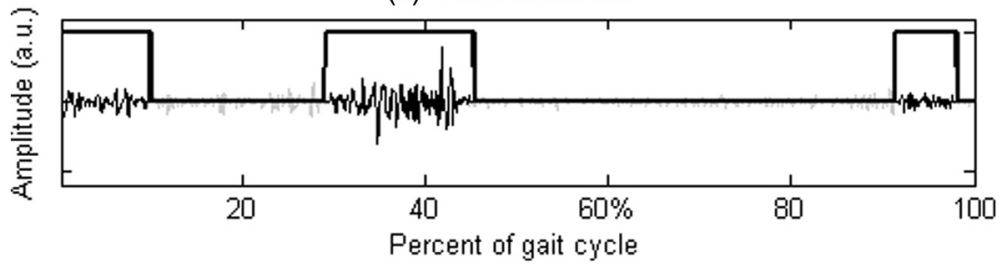

Fig. 3 EMG signal: detection of muscle activation intervals. Examples of gastrocnemius lateralis activation patterns in three different strides of the same subject (left lower limb), showing (a) one, (b) two and (c) three activation intervals within the gait cycle 
"representativeness" of each activation modality, it was calculated its occurrence frequency, i.e. in how many strides a specific modality was observed with respect to the total number of strides. The muscle activation timing over the population was evaluated separately for each activation modality. The number of subjects showing muscle activity at each specific percent of the gait cycle was gray-level coded, with "black" meaning that all subjects showed muscle activity and "white" meaning that none of the subjects activated the muscle. $\mathrm{Matlab}^{\oplus}$ custom routines were used to process the data.

\section{Statistical analysis}

All data distributions were tested for normality with a Kolmogorov-Smirnov test. For each of them, the null hypothesis could not be rejected at a significance level $\alpha=0.05$. For each spatio-temporal and kinematic parameter, a paired $t$-test ( $\alpha=0.05,2$ tails) was applied to determine if there was a significant difference between the conditions of "walking" and "walking while texting". To compare EMG timing between conditions we used 1-way MANOVA approach (Wilk's Lambda statistics): for each muscle, we considered as dependent variables the onset and offset instants of each activation interval, in each modality. Post hoc univariate analysis was performed with $\mathrm{t}$ tests ( $\alpha=0.05,2$ tails) when the MANOVA outcome was significant $(p<0.05)$, to explore in which modality and for which specific activation interval there was a difference between conditions.

\section{Results}

All subjects except one typed the message using both hands. The average typing speed was $80 \pm 13$ characters/minute.

\section{Spatio-temporal parameters}

Texting while walking slowed subjects' gait speed (Table 1), reducing both their cadence and stride length. Conversely, the double support period and $\mathrm{CV}$ of stride time increased. For what concerns the duration of the sub-phases of stance, the flat foot contact increased, and the push-off decreased. Although all the mentioned differences between single-task and dual-task conditions are significant, the absolute effect size is small. In particular, focusing on the variables characterizing gait stability, it can be noticed that the double support period changed only by $2 \%$ GC under dual-task condition, and the $\mathrm{CV}$ of stride time by $0.5 \%$.

\section{Ankle and knee kinematics}

The joint kinematics of the two test conditions were very similar (Fig. 4). Visually, they were practically superimposed at initial contact. A slightly increased ankle dorsi-flexion followed by a slightly reduced plantar-flexion in the "walking and texting" condition could be noticed, but differences in kinematic peak values, always smaller than 2 deg, were never statistically significant (see Table 2).

\section{Muscle activation patterns}

There were no significant differences between single and dual-task conditions, except for the left GL muscle (MANOVA $p=0.02$ ). The post hoc analysis showed that, in the 1-activation modality, the muscle activation onset was delayed under dual-task $(21 \pm$ $6.4 \% \mathrm{GC}$ vs. $16.4 \pm 7.6 \% \mathrm{GC}, p<0.001)$. A pictorial representation of the muscle activation patterns,

Table 1 Gait parameters in single-task and dual-task conditions, and dual-task effect

\begin{tabular}{|c|c|c|c|c|}
\hline & $\begin{array}{l}\text { Walking } \\
\text { (single task) }\end{array}$ & $\begin{array}{l}\text { Walking and texting } \\
\text { (dual task) }\end{array}$ & $p$-value & $\mathrm{DTE}^{\mathrm{b}}$ \\
\hline \multicolumn{5}{|l|}{ Spatio-temporal parameters } \\
\hline Gait speed (m/s) & $1.30 \pm 0.12$ & $1.17 \pm 0.10$ & $<0.001$ & $10.0 \pm 3.8 \%$ \\
\hline Cadence (strides/min) & $54.9 \pm 2.9$ & $52.4 \pm 3.9$ & $<0.001$ & $4.6 \pm 3.1 \%$ \\
\hline Stride length (m) & $1.42 \pm 0.14$ & $1.34 \pm 0.11$ & $<0.001$ & $5.6 \pm 3.5 \%$ \\
\hline Double support (\% GC) & $11.2 \pm 2.7$ & $13.3 \pm 2.3$ & $<0.001$ & $23 \% \pm 20 \%$ \\
\hline \multicolumn{5}{|l|}{ Stride-to-stride variability } \\
\hline $\mathrm{CV}^{\mathrm{a}}$ of stride time (\%) & $1.86 \pm 0.42$ & $2.33 \pm 0.63$ & 0.008 & $28 \pm 34 \%$ \\
\hline \multicolumn{5}{|l|}{ Sub-phases of stance (duration) } \\
\hline H, Heel contact (\% GC) & $6.6 \pm 2.0$ & $6.9 \pm 3.3$ & 0.4 & - \\
\hline F, Flat foot contact (\% GC) & $26.4 \pm 4.0$ & $30.0 \pm 4.3$ & $<0.001$ & $14 \pm 8 \%$ \\
\hline P, Push off (\% GC) & $22.6 \pm 4.0$ & $19.8 \pm 3.4$ & $<0.001$ & $12 \pm 6 \%$ \\
\hline
\end{tabular}

Values are mean \pm standard deviation over the population. The left and right side values were averaged

${ }^{\mathrm{a}} \mathrm{CV}$ : Coefficient of Variation $=($ standard deviation $/$ mean $) \times 100$

${ }^{\mathrm{b}}$ DTE: Dual Task Effect $=[\mid($ single-task - dual-task) $\mid /$ single-task $] \times 100$ 

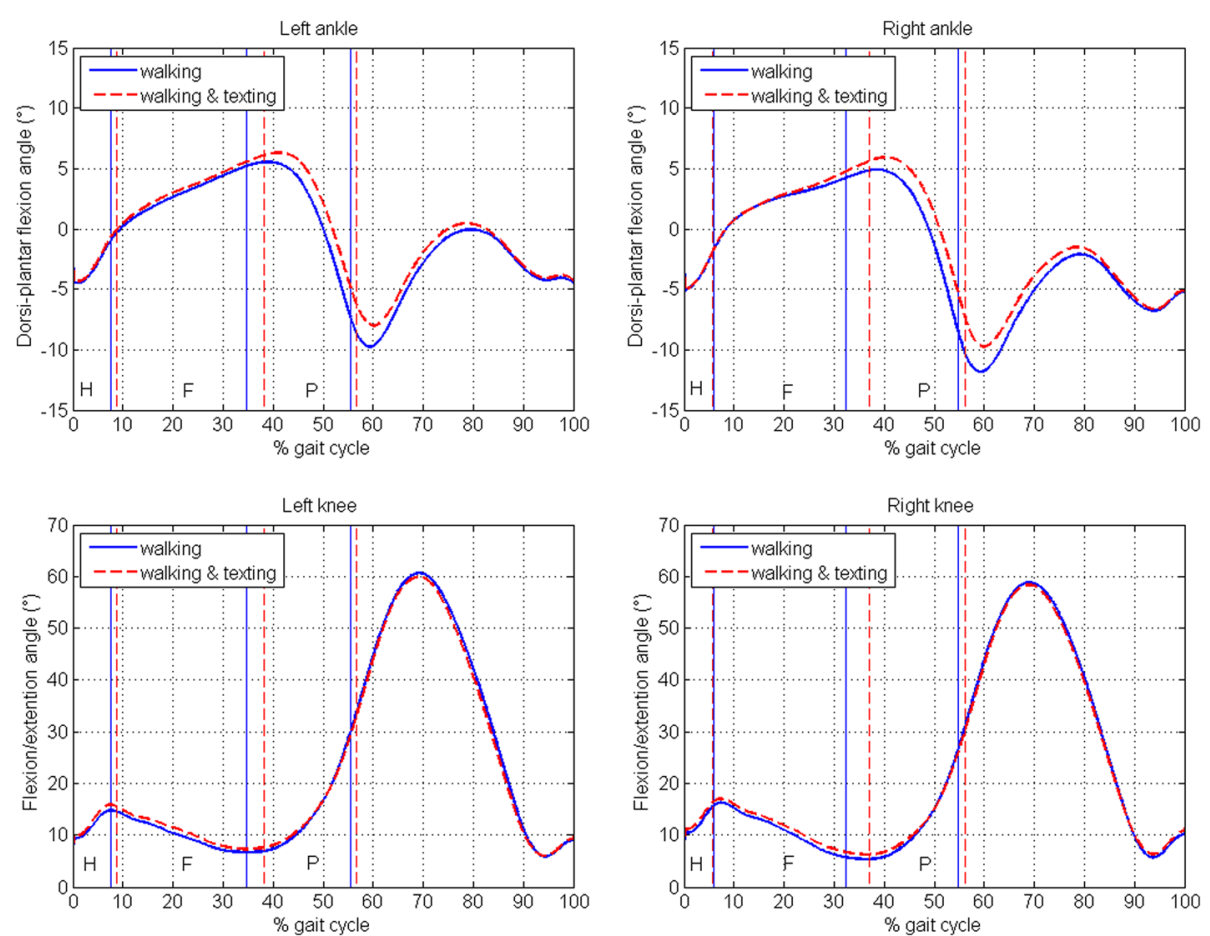

Fig. 4 Ankle and knee joint kinematics. Ankle and knee joint kinematics for the left and right side are represented (multiple strides were averaged for each subject and then the global average across subjects was considered). Two conditions are depicted: walking (blue continuous line) and walking \& texting (red dashed line). The sub-phases of stance ( $\mathrm{H}$ : heel contact, F: flat-foot contact, P: push-off) are delimited by vertical lines for both conditions

obtained separating the different activation modalities, was reported in Fig. 5.

\section{Ankle muscles co-contraction}

Under dual-task, the co-contraction of TA and GL was augmented in some of the sub-phases of stance, and it was diminished in others. More specifically, during the $\mathrm{H}$-phase, the percentage of cycles showing cocontraction was augmented, although the statistical significance was not reached (Fig. 6). In these cycles,

Table 2 Kinematic angles

\begin{tabular}{|c|c|c|c|}
\hline & $\begin{array}{l}\text { Walking } \\
\text { (single task) }\end{array}$ & $\begin{array}{l}\text { Walking and texting } \\
\text { (dual task) }\end{array}$ & $p$-value \\
\hline \multicolumn{4}{|l|}{ Ankle } \\
\hline $\begin{array}{l}\text { Max dorsi-plantar } \\
\text { flexion }\left(^{(}\right)\end{array}$ & $5.4 \pm 2.3$ & $6.3 \pm 3.1$ & 0.39 \\
\hline $\begin{array}{l}\text { Min dorsi-plantar } \\
\text { flexion }\left(^{\circ}\right)\end{array}$ & $-11.1 \pm 4.8$ & $-9.2 \pm 5.1$ & 0.35 \\
\hline \multicolumn{4}{|l|}{ Knee } \\
\hline $\begin{array}{l}\text { First peak of knee } \\
\text { flexion }\left(^{\circ}\right)\end{array}$ & $15.7 \pm 4.9$ & $16.6 \pm 4.9$ & 0.66 \\
\hline $\begin{array}{l}\text { Max of knee } \\
\text { flexion }\left(^{\circ}\right)\end{array}$ & $60.0 \pm 5.5$ & $59.4 \pm 6.0$ & 0.81 \\
\hline
\end{tabular}

Values are mean \pm standard deviation over the population. The left and right side values were averaged the co-contraction duration was slightly increased (from 3.4 to $3.6 \%, p=0.03$ ). During the F-phase, the percentage of cycles showing co-contraction was augmented (from 49.4 to $59.4 \%, p<0.001$ ). Also the cocontraction duration was increased (from 7.2 to $8.1 \%$, $p<0.001)$. During the $\mathrm{P}$-phase, the percentage of cycles showing co-contraction was diminished (from 44.3 to $38.2 \%, p=0.04$ ). Also the co-contraction duration was diminished (from 6.1 to $4.8 \%, p<0.001$ ). In swing, there were no significant changes in the TA/ GL co-contractions.

\section{Discussion}

\section{Spatio-temporal parameters}

The task assigned to participants involved both "thinking" and "typing" while walking, as it happens in the everydaylife use of a smartphone. Walking-typing most probably increased the visuospatial attentional load, while walkingthinking allowed the participant to spend more time looking at the path instead of the display. This might explain the small velocity reduction observed. On the average, young adults slowed their gait speed only by $10 \%$ when texting while walking. In literature, it was reported a reduction of $23 \%$ when typing a phrase appearing on the smartphone screen [6] and a reduction of $32 \%$ when typing a pre-assigned sentence [3]. On the other hand, it 


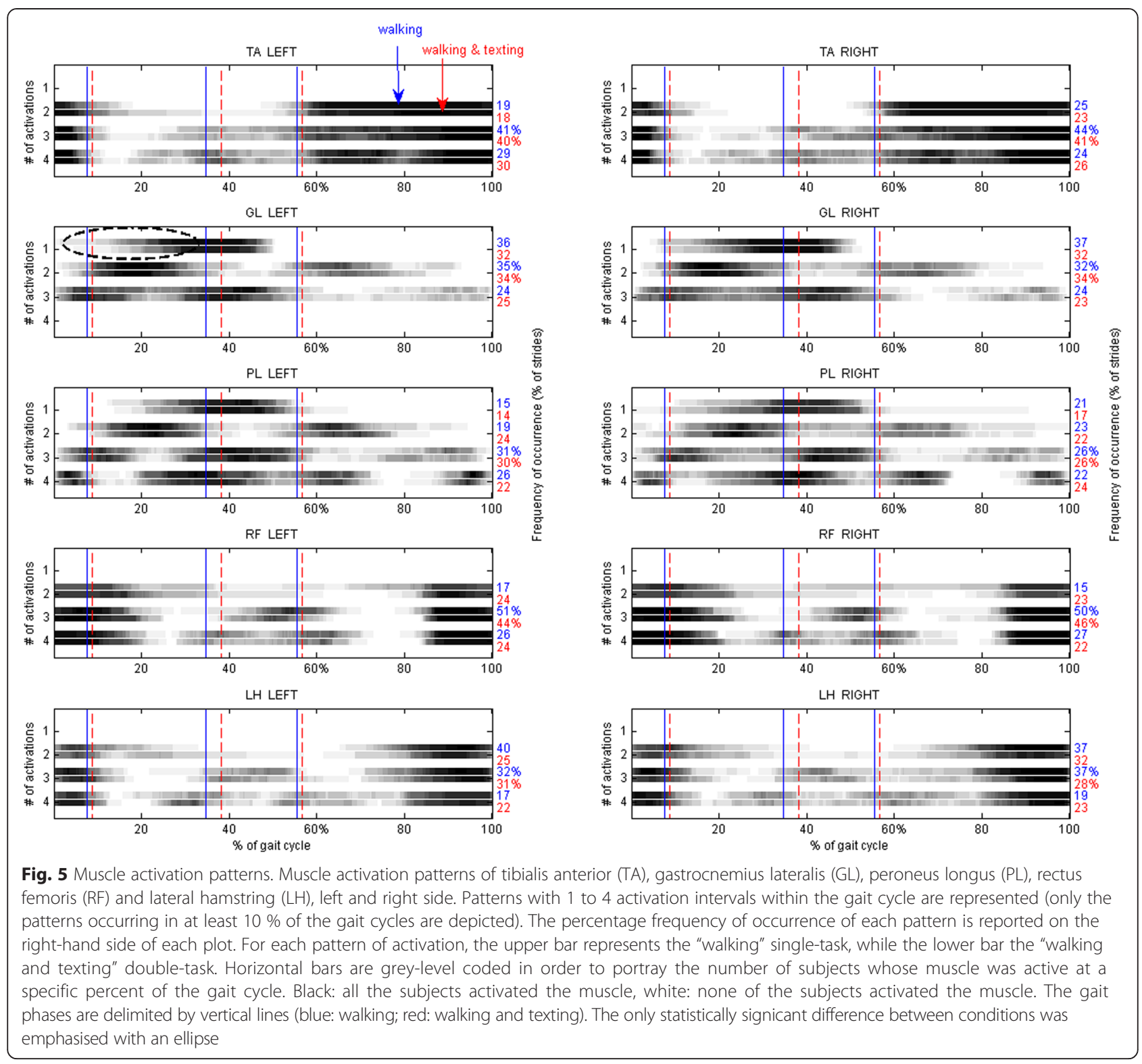

was reported a reduction of $17 \%$ when writing an email while walking, answering a question previously posed [22], a protocol more similar to the one we used, in that it implies also "thinking" and not only "typing". However, differently from [22], we analyzed a prolonged task lasting $3 \mathrm{~min}$ along a $15-\mathrm{m}$ walkway, instead of 3 separate $10-\mathrm{m}$ trials. It was not possible to establish if participants were writing at the same typing speed throughout, but we checked that they maintained a stable gait speed among the walkway passages.

The average typing speed that we obtained was much slower $(80 \pm 13$ chars $/ \mathrm{min}$.) than in other studies $(222 \pm$ 45 chars/min) [6]. This is not surprising since the secondary task (texting) was different. In [6] participants were instructed to type the phrase that appeared on the screen "as quickly and as accurate as possible into the textbox below the phrase", while our participants were engaged also in a memory effort when asked to describe their activity on the day before the test. Therefore, the slower typing speed may be explained by the fact that we did not chose a pure "typing" task (like typing a predetermined sentence as fast as possible), but a more realistic condition in which the subject also had to think to what he was writing. This slowed the typing speed, but limited to a small amount the gait speed decrease under dual-task (10\%).

Furthermore, our results showed an increase in stride time variability under a dual-task $(28 \%)$ higher than that reported $(17 \%)$ when analyzing a pure cognitive task (backward counting) [8]. Again this is not surprising 


\section{(a) Cycles showing co-contraction}

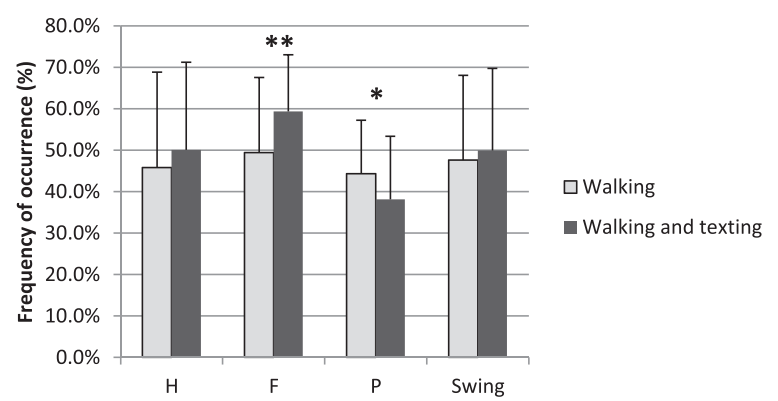

(b) Co-contraction duration

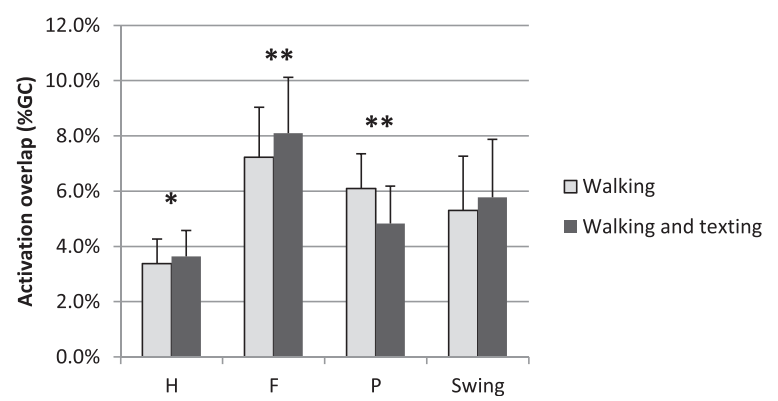

Fig. 6 Co-contraction of ankle antagonist muscles. Co-contraction between tibialis anterior (TA) and gastrocnemius lateralis (GL). (a) Percentage of cycles with TA/GL co-contraction during heel contact $(H)$, flat foot contact $(F)$, push off $(P)$ and swing. (b) Co-contraction duration (expressed as \% of gait cycle). Significant differences between walking conditions are indicated as $*(p<0.05)$ or ** $(p<0.001)$

since the task that we considered involved not only cognitive resources, but also the integrated use of near and far vision and bimanual coordination.

For what concerned the sub-phases of stance, our results showed that the F-phase was prolonged by 3.6 $\% \mathrm{GC}$ and that the P-phase was shortened by $2.8 \% \mathrm{GC}$, under dual-task. These small changes may be explained by the gait speed (and stride length) reduction.

\section{Ankle and knee kinematics}

Our results did not reveal any significant alterations of the ankle and knee joint kinematics.

\section{Muscle activation patterns}

The muscle activation patterns did not show statistically significant modifications when texting while walking, with the exception of a slightly delayed onset of the left $\mathrm{GL}$, in the first activation modality.

\section{Ankle muscle co-contraction}

Co-contraction is a strategy used by the CNS to achieve movement accuracy by controlling dynamic joint stability, especially during the learning process of a novel task [23-25]. However, the majority of the studies about the role of co-contraction on human motor control focused their attention on the upper limb [26]. Our results showed that the ankle muscle co-contractions were slightly augmented in the $\mathrm{H}$-phase (roughly corresponding to load response) and in the F-phase (mid-stance), when the foot reached the full contact with the floor initiating the single limb stance. Conversely, the co-contractions decreased during the P-phase (terminal stance).

Our results may be interpreted as an increased need of stabilizing the ankle joint during a "critical" phase of the gait cycle, when the body weight was transferred from one leg to the other. The decrease of co-contractions in terminal stance may indicate that the CNS supplied more "attention" to the contralateral limb on whom the weight load was being transferred. Hence, the motor control strategy seemed different in the different phases of the gait cycle: increasing co-contractions when the body load was sustained by a single limb; decreasing cocontractions when both feet were providing a proprioceptive input. This finding was probably not influenced by the walking speed reduction. In fact, previous research demonstrated no modifications in the ankle muscle co-contraction levels when reducing the walking speed by $10 \%$ [27].

Globally, there weren't any evident trends in data suggesting that those who typed faster (i.e. those that could be argued to be more attentional loaded with the texting task) had larger gait DTE. In cognitive sciences is being debated the concept of "digital natives" [28] to indicate young individuals that have spent their entire lives surrounded by the tools of the digital age, naturally skilled at multitasking. While the concept is new in the field of gait analysis, our results seem to indicate that, overall, the gait modifications due to texting while walking are minimal in young adults. However, we do not interpret our results to mean that texting while walking is a "safe" dual task activity. Safe ambulation in the real world requires appropriate attentional resources to maintain dynamic stability while monitoring for environmental hazards $[4,29]$ and the difference between laboratory and real-world settings are well documented [5].

\section{Study limitations}

It is very difficult to identify if the effects of texting while walking are due to changes in gait speed between the conditions, or if they are due to the effects of texting. We had no control conditions in which the walking speed was matched. Hence, we cannot exclude that the findings that we obtained could be explained solely by the change in walking speed. Nevertheless, there are no clear trends indicating that participants who reduced more their walking speed showed a correspondingly higher co-contraction increase. 
We measured only the average typing speed, and hence we do not know if the participants were writing at the same typing speed throughout. Furthermore, we had no measure of the time participants spent walking-typing vs. walking-thinking. This could be important since walking-thinking would result in more time looking at the path. This evaluation could also be addressed by taking some measure of eye movements to estimate time spent looking at screen vs. path. Future studies may consider including mobile eye-tracker devices to this purpose.

\section{Conclusions}

Young adults engaged in the double task of texting while walking showed minimal modifications to their walking scheme. They slightly reduced their gait speed to safely cope with the task. Gait adaptations in terms of 1) subphases of stance, 2) stride-to-stride variability, 3) ankle and knee joint kinematics, 4) muscle activation patterns, and 5) co-contraction of ankle antagonist muscles were comprehensively documented for the first time. We found an increased co-contraction of the ankle antagonist muscles in the "critical" gait phase spanning from load response to mid-stance, phase that corresponds to the body weight transfer from one leg to the other. This seems a CNS adaptation under dual task, responding to an increased need for ankle stabilization.

The methodology described to study the muscle activation patterns and co-contractions by means of statistical gait analysis may be extended to other dual-task studies.

\section{Abbreviations \\ CNS: Central nervous system; CV: Coefficient of variation; DTE: Dual task effect; EMG: Electromyography; F: Flat-foot contact; GC: Gait cycle; GL: Gastrocnemius lateralis; H: Heel contact; LH: Lateral hamstring; P: Push off; PL: Peroneus longus; RF: Rectus femoris; TA: Tibialis anterior.}

\section{Competing interests}

The authors declare that they have no competing interests.

\section{Authors' contributions}

All authors have given final approval of the version to be published and agree to be accountable for all aspects of the work in ensuring that questions related to the accuracy or integrity of any part of the work are appropriately investigated and resolved. Author V.A. has contributed to the study conception and design, acquisition of data, analysis and interpretation of data, and has drafted the manuscript. Author F.L.F. has contributed to the study conception and design, acquisition of data and analysis of data, and has critically revised the manuscript. Author G.M. has contributed to the study conception and design and has critically revised the manuscript. Author M.K. has contributed to the study conception and design, analysis and interpretation of data and has critically revised the manuscript.

\section{Acknowledgements}

This work was supported by the contract number 355/2012 between Azienda Ospedaliera Univ. (A.O.U.) San Giovanni Battista di Torino and Politecnico di Torino. The A.O.U. San Giovanni Battista di Torino played no role in the design, collection, analysis, and interpretation of data, in the writing of the manuscript, and in the decision to submit the manuscript for publication.

\section{Author details}

'Dipartimento di Elettronica e Telecomunicazioni, Politecnico di Torino, Corso Duca degli Abruzzi 24, 10129 Torino, Italy. ${ }^{2}$ Università degli Studi di Torino, Via Zuretti 29, 10126 Torino, Italy. ${ }^{3}$ SC Medicina Fisica e Riabilitazione U, AO Città della Salute e della Scienza, Corso Bramante 82, 10126 Torino, Italy.

Received: 20 March 2015 Accepted: 17 September 2015

Published online: 22 September 2015

\section{References}

1. Nasar J, Hecht P, Wener R. Mobile telephones, distracted attention, and pedestrian safety. Accid Anal Prev. 2008;40(1):69-75.

2. Lamberg EM, Muratori LM. Cell phones change the way we walk. Gait Posture. 2012;35(4):688-90. Erratum in. Gait Posture. 2012;36(3):655.

3. Schabrun SM, van den Hoorn W, Moorcroft A, Greenland C, Hodges PW. Texting and walking: strategies for postural control and implications for safety. PLoS One 2014;9(1):e84312. Erratum in. PLoS One. 2014;9(2), e91489.

4. Marone JR, Patel PB, Hurt CP, Grabiner MD. Frontal plane margin of stability is increased during texting while walking. Gait Posture. 2014;40(1):243-6.

5. Kao $\mathrm{P}$, Higginson $\mathrm{Cl}$, Seymour K, Kamerdze M, Higginson JS. Walking stability during cell phone use in healthy adults. Gait Posture. 2015;41:947-53.

6. Plummer P, Apple S, Dowd C, Keith E. Texting and walking: Effect of environmental setting and task prioritization on dual-task interference in healthy young adults. Gait Posture. 2015:41(1):46-51.

7. Parr ND, Hass CJ, Tillman MD. Cellular Phone Texting Impairs Gait in Able-Bodied Young Adults. J Appl Biomech. 2014;30(6):685-8.

8. Beauchet O, Dubost V, Herrmann FR, Kressig RW. Stride-to-stride variability while backward counting among healthy young adults. J Neuroeng Rehabil. 2005;2:26 [Open access: http://dx.doi.org/10.1186/ 1743-0003-2-26]

9. Yogev-Seligmann G, Rotem-Galili Y, Mirelman A, Dickstein R, Giladi N, Hausdorff JM. How Does Explicit Prioritization Alter Walking During Dual-Task Performance? Effects of Age and Sex on Gait Speed and Variability. Phys Ther. 2010;90:177-86.

10. Dubost V, Annweiler C, Aminian K, Najafi B, Herrmann FR, Beauchet O. Stride-to-stride variability while enumerating animal names among healthy young adults: Result of stride velocity or effect of attention-demanding task? Gait Posture. 2008:27:138-43.

11. Agostini $V$, Knaflitz M. Statistical gait analysis. In: Acharya RU, Molinari F, Tamura T, Naidu DS, Suri JS, editors. Distributed Diagnosis and Home Healthcare $\left(\mathrm{D}_{2} \mathrm{H}_{2}\right)$. Vol. 2. Stevenson Ranch: American Scientific Publishers; 2012. p. 99-121.

12. Agostini $V$, Nascimbeni A, Gaffuri A, Imazio P, Benedetti MG, Knaflitz M. Normative EMG activation patterns of school-age children during gait. Gait Posture. 2010;32:285-9.

13. Agostini V, Ganio D, Facchin K, Cane L, Moreira Carneiro S, Knaflitz M. Gait parameters and muscle activation patterns at 3,6 and 12 months after Total Hip Arthroplasty. J Arthroplasty. 2014;29(6):1265-72.

14. Benedetti MG, Agostini V, Knaflitz M, Gasparroni V, Boschi M, Piperno R. Self-reported gait unsteadiness in mildly impaired neurological patients: an objective assessment through statistical gait analysis. J Neuroeng Rehabil. 2012:9:64 [Open access: doi:10.1186/1743-0003-9-64].

15. Agostini V, Nascimbeni A, Gaffuri A, Knaflitz M, Gait measurements in hemiplegic children: an automatic analysis of foot-floor contact sequences and electromyographic patterns. Proc. USA: IEEE International Symposium on Medical Measurements and Applications (MeMea); 2014.

16. Olney SJ. Quantitative evaluation of cocontraction of knee and ankle muscles in normal walking. Biomechanics, international series on biomechanics, ix-a. Champaign (IL): Human Kinetics Publishers; 1985. p. 431-5.

17. Di Nardo F, Mengarelli A, Maranesi E, Burattini L, Fioretti S. Assessment of the ankle muscle co-contraction during normal gait: A surface electromyography study. J Electromyogr Kinesiol. 2014;25(2):347-54.

18. Yasuda $\mathrm{K}$, Sato $\mathrm{Y}$, limura $\mathrm{N}$, Iwata $\mathrm{H}$. Allocation of attentional resources toward a secondary cognitive task leads to compromised ankle proprioceptive performance in healthy young adults. Rehabil Res Pract. 2014;170304:1-7 [Open access: http://dx.doi.org/10.1155/2014/170304].

19. Agostini $V$, Balestra $G$, Knaflitz M. Segmentation and classification of gait cycles. IEEE Trans Neural Syst Rehabil Eng. 2014;22(5):946-52. 
20. Bonato P, D'Alessio T, Knaflitz M. A statistical method for the measurement of muscle activation intervals from surface myoelectric signal during gait. IEEE Trans Biomed Eng. 1998;45:287-99.

21. Agostini $V$, Knaflitz M. An algorithm for the estimation of the Signal-To-Noise ratio in surface myoelectric signals generated during cyclic movements. IEEE Trans Biomed Eng. 2012;59(1):219-25.

22. Demura S, Uchiyama M. Influence of cell phone email use on characteristics of gait. Eur J Sport Sci. 2009;9(5):303-09.

23. De Luca CJ, Mambrito B. Voluntary control of motor units in human antagonist muscles - coactivation and reciprocal activation. J Neurophysiol. 1987;58:525-42.

24. Osu R, Franklin DW, Kato H, Gomi H, Domen K, Yoshioka T, et al. Short and long-term changes in joint co-contraction associated with motor learning as revealed from surface EMG. J Neurophysiol. 2002;88:991-1004.

25. Gribble PL, Mullin LI, Cothros N, Mattar A. Role of cocontraction in arm movement accuracy. J Neurophysiol. 2003;89:2396-405.

26. Rosa M. Co-contraction role on human control. A neural basis. J Nov Physiother. 2015;5:1 [Open access: doi:10.4172/2165-7025.1000248]

27. Seyedali M, Czerniecki JM, Morgenroth DC, Hahn ME. Co-contraction patterns of trans-tibial amputee ankle and knee musculature during gait. J Neuroeng Rehabil. 2012;9:29 [Open access: doi:10.1186/1743-0003-9-29].

28. Prensky M. Digital Natives, Digital Immigrants Part 1. On the Horizon. $2001 ; 9(5): 1-6$.

29. Thompson LL, Rivara FP, Ayyagari RC, Ebel BE. Impact of social and technological distraction on pedestrian crossing behaviour: an observational study. Inj Prev. 2013;19:232-37.

\section{Submit your next manuscript to BioMed Central and take full advantage of:}

- Convenient online submission

- Thorough peer review

- No space constraints or color figure charges

- Immediate publication on acceptance

- Inclusion in PubMed, CAS, Scopus and Google Scholar

- Research which is freely available for redistribution 